\section{Commentary: A long road back to the beginning}

\author{
David D'Alessandro, MD, and \\ Richard N. Pierson III, MD
}

Use of donation after circulatory death (DCD) hearts could significantly extend cardiac transplantation's therapeutic reach. Smith and colleagues ${ }^{1}$ should be congratulated on their pioneering work in which they report the first successful use of the normothermic regional perfusion (NRP) technique in an adult DCD heart program in the United States. These authors describe an innovative protocol that relocated potential donor patients to the New York University Medical Center for the purpose of organ donation. Eight hearts were recovered and successfully transplanted with $100 \%$ 30-day recipient survival. Ironically, this latest advancement in our field returns us to the very first heart transplant, performed by Christian Barnard more than 50 years ago using a heart from a DCD donor who was resuscitated on cardiopulmonary bypass. ${ }^{2}$

Once brain death criteria were established and legally recognized worldwide, the use of brain dead, heartbeating donors became the standard practice, with outstanding, highly reproducible results. The ethical principles involved are well established and for the most part not controversial. The ethical principles of DCD organ transplantation, in contrast, are not as firmly established. Donor management practices and local governances related to DCD organ donation vary considerably between hospitals, both within the United States and across the globe. In an effort to address these and related concerns, an expert consensus document was recently published that addresses

\footnotetext{
From the Division of Cardiac Surgery, Department of Surgery, Massachusetts General Hospital, Boston, Mass.

Disclosures: The authors reported no conflicts of interest.

The Journal policy requires editors and reviewers to disclose conflicts of interest and to decline handling or reviewing manuscripts for which they may have a conflict of interest. The editors and reviewers of this article have no conflicts of interest.

Received for publication July 29, 2021; revisions received July 29, 2021; accepted for publication Aug 3, 2021; available ahead of print Aug 8, 2021.

Address for reprints: David D'Alessandro, MD, Division of Cardiac Surgery, Department of Surgery, Massachusetts General Hospital, 55 Fruit St, Cox Bldg, Room 644, Boston, MA 02114 (E-mail: dadalessandro@mgh.harvard.edu).

J Thorac Cardiovasc Surg 2022;164:569-70

$0022-5223 / \$ 36.00$

Copyright (c) 2021 by The American Association for Thoracic Surgery

https://doi.org/10.1016/j.jtcvs.2021.08.012
}

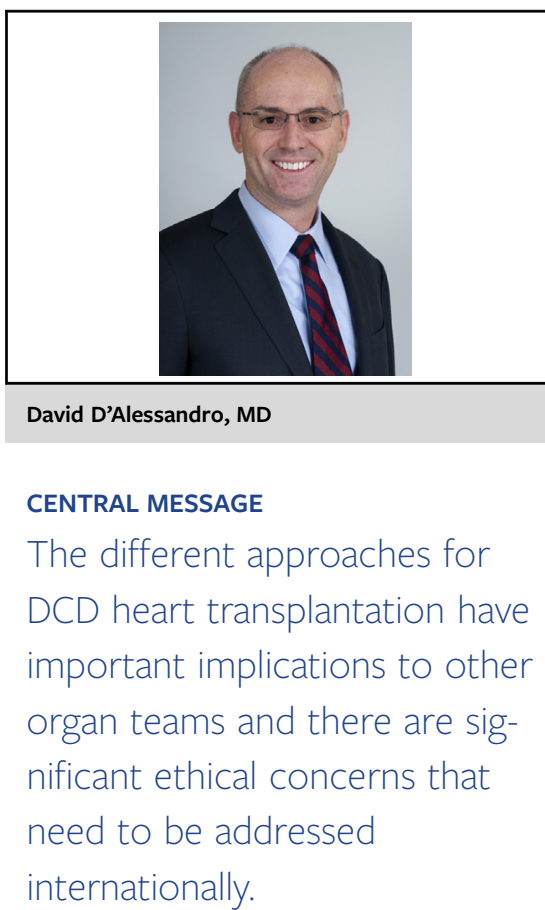

many of the fundamental issues and provides a thoughtful framework for discussion.

Two alternative heart resuscitation techniques have been demonstrated to allow consistently successful use of DCD heart allografts. The ex vivo approach involves the removal of the heart following determination of circulatory death. Resuscitation and reanimation of the heart utilizes a proprietary ex vivo perfusion apparatus primed with donor blood, and depends on a biochemical surrogate, cardiac lactate consumption, to predict adequate cardiac function in the recipient. This technique has been performed in the United States only in the confines of an ongoing clinical trial (ClinicalTrials.gov identifier: NCT03831048). Since that study began in December 2019, 180 patients have undergone transplant in the trial and the continued access protocol, which is now closed. The rapid enrollment into this trial demonstrates the clinical appetite for additional hearts to meet clinical demand, and the feasibility and scalability of this approach.

The in situ heart resuscitation technique reported herein utilized central cannulation and cardiopulmonary bypass to reestablish warm systemic perfusion after excluding the donor's cerebral circulation. As described by the authors, this approach offers some advantages, including, most importantly, allowing the controlled resuscitation and evaluation of all the donor's organs in situ. The reestablishment 
of donor blood flow has been a source of ethical concern. Although the technique includes clamping the arch vessels before reestablishing donor systemic circulation, critics have pointed out that this approach does not take into account unexpected anatomic anomalies, nor prevent collateral flow to the brain stem or even to the intracranial circulation. (A recent position paper advocates for opening the aortic arch vessels distally to vent them to atmospheric pressure to prevent this unlikely but ethically troublesome possibility. ${ }^{4}$ This maneuver was not performed in the New York University series.) Further, there is justifiable concern that the NRP technique could conflict with the US Uniform Determination of Death Act, which in the absence of brain death, determines death on the basis of "irreversible cessation of circulatory and respiratory functions. ${ }^{5}$ In contrast, the ex vivo heart resuscitation approach, while requiring costly equipment and specialized technical expertise, is less ethically controversial because the risk of inadvertent restoration of brain stem or intracranial blood flow is avoided.

The practice of transplantation is made possible by the altruistic participation of a trusting population. We must fiercely protect our ethical standards and our Hippocratic Oath for all the patients we are privileged to care for, including nonbrain-dead potential heart donors. In our opinion, this imperative includes not subjecting potential donors to invasive procedures such as coronary catheterizations for the sole purpose evaluating a patient's heart for transplant, as described by these authors. Also of concern, the act of transporting potential donors to the recipient's hospital removes these patients from the care of their local practitioners, takes them further from their families, and blurs the line that separates transplant programs from participating in the care of donors who are not deceased. Our system needs to protect the most vulnerable, including those who may not have medical guardians who exhibit the rigor, tenacity, and ethical probity that we surgeons take pride in.
As a global transplant community, we must critically examine our evolving practices. In pushing the DCD boundaries, as Smith and colleagues ${ }^{1}$ have proposed, we need to ensure that we do not erode the public trust we have worked so hard to secure. Our overarching concern is that an unchecked push forward may result in a precipitous fall backward, as would occur if a recent proposal for a moratorium on NRP DCD programs were to gain traction (https://www.acponline.org/acp_policy/policies/ethics_ determination_of_death_and_organ_transplantation_in_ nrp_2021.pdf). The pioneers among us must choose not to skirt ethical boundaries or ignore legitimate concerns raised by experts or the general public.

More generally, we believe that medical ethics should not vary from 1 medical center to another, or from 1 region or country to another. We in medicine and surgery have a responsibility to participate in defining best practices and to defend what is right. In our fully justifiable efforts to increase access to scarce donor hearts, let us be sure we are not taking a wrong turn. If we are thoughtful and deliberate, it will become clear whether in situ heart resuscitation is in fact a step forward on the long road ahead.

\section{References}

1. Smith DE, Kon ZN, Carillo JA, Chen S, Gidea CG, Piper GL, et al. Early experience with donation after circulatory death heart transplantation using normothermic regional perfusion in the United States. J Thorac Cardiovasc Surg. 2022;164:557-68.e1.

2. Barnard $\mathrm{CN}$. The operation. A human cardiac transplant: an interim report of a successful operation performed at Groote Schuur Hospital, Cape Town. S Afr Med J. 1967;41:1271-4.

3. Dominguez-Gil B, Ascher N, Capron AM, Gardiner D, Manara AR, Bernat JL, et al. Expanding controlled donation after the circulatory determination of death: statement from an International Collaborative. Intensive Care Med. 2021;47:265-81.

4. Manara A, Shemie SD, Large S, Healey A, Baker A, Badiwala M, et al. Maintaining the permanence principle for death during in situ normothermic regional perfusion for donation after circulatory death organ recovery: a United Kingdom and Canadian proposal. Am J Transplant. 2020;20:2017-25.

5. US Uniform Determination of Death Act. Available at: https://www.uniformlaws. org/HigherLogic/System/DownloadDocumentFile.ashx? DocumentFileKey=341 343fa-1efe-706c-043a-9290fdcfd909\&forceDialog=0. Accessed September 4, 2021. 